BULL. AUSTRAL. MATH. SOC.

VOL. $5(197 \mid), 391-410$.

\title{
Varieties of soluble groups and a dichotomy of P. Hall
}

\author{
J.R.J. Groves
}

\begin{abstract}
Let $\stackrel{A}{=}$ denote the variety of all abelian groups and, for each prime $p$, let $\stackrel{A}{\Rightarrow}$ be the variety of all elementary abelian p-groups. Let $\underline{\underline{V}}$ be a subvariety of a product of (finitely many) varieties each of which is either soluble or Cross. The results of this paper are the following.
\end{abstract}

(i) If $\underline{\underline{V}}$ contains no $\stackrel{\underline{\underline{A A}}}{p}$, then $\underline{\underline{\mathrm{V}}}$ is finite exponent by nilpotent.

(ii) If $\underline{\underline{V}}$ contains no $\underline{\underline{A}} \underline{\underline{A}}$, then $\underline{\underline{V}}$ is nilpotent by finite exponent.

(iii) If $\underline{\underline{V}}$ contains no $\underline{\underline{A} \underline{A}}$ and no $\underline{\underline{A A}} p$, then $\underline{\underline{V}}$ is the join of a nilpotent variety and a variety of finite exponent.

(iv) If $\underline{\underline{V}}$ does not contain ( $\operatorname{var} G) \underline{\underline{A}}$ for any nonabelian finite simple group $G$, then $\underline{V}$ is soluble by finite exponent.

For soluble $\underline{\underline{V}}$, the results (i)-(iii) are shown to extend a dichotomy discussed by $P$. Hall.

\section{Introduction}

In this paper, we prove a number of results on varieties of groups.

Received 13 July 1971. This work was done towards a doctoral thesis at the Australian National University. The author thanks his supervisor, Dr L.G. Kovács, for his invaluable help and encouragement. 
These are 'external results' in the sense of Kovács and Newman [12]; that is, they take the form 'if $\underline{\underline{V}}$ is a variety with no subvariety from a class $w$, then ...'. We also show how our results contribute to giving an extension of a dichotomy of P. Hall.

By means of commutator-subgroup functions, Hall defined a special class of varieties of groups - the class of all varieties obtainable from the trivial variety by commutation. He then showed that certain natural dichotomies of this class can be used to distinguish properties of soluble groups (see [5; pp. 422-423], [7; pp. 601-603́], [8; pp. 328, 333] for a discussion of these dichotomies).

It is natural to ask whether one may find similar dichotomies of all soluble varieties of groups and whether these also distinguish interesting group theoretical properties. Among other dichotomies, the special class of varieties defined by $\mathrm{Hall}$ splits into those which are nilpotent and those which contain the variety of all metabelian groups. Here we show that there is a reasonably complete set of dichotomies in the class of all soluble varieties which extend this dichotomy.

Before we state our results, we refer to Hanna Neumann's book [14] for notation, terminology and basic results, with the following exceptions: we use doubly underlined Roman capitals, rather than German capitals, for varieties; we use $\underline{\underline{V}}(G)$ for the verbal subgroup of a group corresponding to the variety $\underline{\underline{V}}$; and we do not reserve $G, H$ for relatively free groups nor $F$ for an absolutely free group.

We first state our main theorem in a somewhat restricted form. (This is the version we require to extend the Hall dichotomy. The result appears in full strength as Theorem C.)

THEOREM A. Let $\underline{\underline{\mathrm{V}}}$ be a soluble variety.

(i) If $\stackrel{\underline{\mathrm{AA}}}{\mathrm{p}} \neq \underline{\underline{\mathrm{V}}}$ for all primes $p$, there exist natural numbers $k, Z$ and $c$ such that $\underline{\underline{\mathrm{V}}} \leq \mathrm{A}_{=}^{2} \underline{\mathrm{N}}_{\mathrm{C}}$.

(ii) If $\underline{\underline{A}} \underline{\underline{A}} \neq \mathrm{V}$ for all primes $p$, there exist natural numbers $k, 2$ and $c$ such that $\underline{\underline{\mathrm{V}}} \leq \underline{\underline{\mathrm{N}}} \mathrm{c}_{\mathrm{A}}^{2}$. 
(iii) If $\stackrel{\underline{\mathrm{AA}}}{\underline{\underline{\mathrm{V}}}}$ and $\underline{\underline{\mathrm{A}}} \underline{\underline{\mathrm{A}}} \neq \underline{\underline{\mathrm{V}}}$ for all primes $p$, there exist a nilpotent variety $\underline{N}$ and a variety $\underline{\underline{P}}$ of finite exponent such that $\underline{\underline{\mathrm{V}}}=\underline{\underline{\mathrm{N}}} \vee \underline{\underline{\mathrm{P}}}$.

We note that (iii) follows immediately from (i) and (ii) and

LEMMA A. Let $\mathrm{V}$ be a (not necessarily soluble) variety and suppose that $\underline{\underline{\mathrm{V}}} \leq \underline{\underline{\mathrm{B}}} n^{\mathrm{N}}{ }_{c} \wedge \underline{\underline{\mathrm{N}}} \mathrm{C}_{n}$ for some natural numbers $n$ and $c$. Then there exist a nilpotent variety $\underline{\underline{\mathbb{N}}}$ and a variety $\underline{\underline{\mathrm{P}}}$ of finite exponent such that $\underline{\underline{\mathrm{V}}}=\underline{\underline{\mathrm{N}}} \vee \underline{\underline{\mathrm{P}}}$.

We require two more results for this discussion.

PROPOSITION 1. Let $\mathrm{V}$ be a soluble variety such that

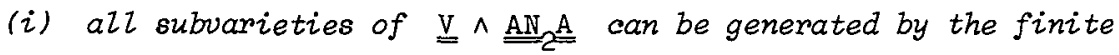
groups they contain.

Then, if $\underline{\underline{V}}$ does not contain $\underline{\underline{A}}^{2}$,

(ii) there exist natural numbers $n$ and $c$ such that $\underline{\underline{\mathrm{v}}} \leq \underline{\mathrm{B}}_{n} \underline{\mathrm{N}} c_{-} \mathrm{B}_{n}$.

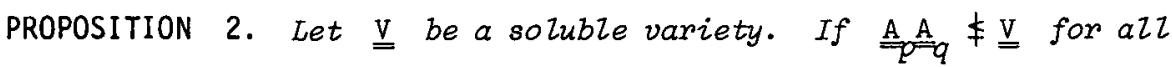
(not necessarily distinct) primes $p$ and $q$, then $\underline{V}$ is nilpotent.

Proposition 1 is proved in [3]. Although Proposition 2 appears to be well known, we are not aware of a proof in the literature and so we sketch one here. Suppose, then, that $\underset{A p}{A} \not \mathrm{A} q \mathrm{~V}$ for $a l l$ primes $p$ and $q$. Firstly, by Theorem A ( $i i i)$, we may suppose that $\underline{\underline{V}}$ has finite exponent. Thus, since it is soluble, $\underline{\underline{V}}$ is locally finite and so can be generated by its finite groups. However, as Kovács and Newman have noted $[11 ; \mathrm{p} .222]$, a nonnilpotent finite group of least order in $\underline{\underline{V}}$ would generate an $\stackrel{A}{\Rightarrow} q$, and so all the finite groups of $\underline{\underline{V}}$ must be nilpotent. But by a result of the same authors [12; Theorem 5], there is a bound on the class of the nilpotent groups in $\underline{\underline{V}}$, since $\underline{A} \underline{\underline{A}} p \neq \underline{\underline{V}}$ for all primes $p$. The proposition follows.

Although we have stated these results as external results - in which form they naturally arose - rather than as dichotomies, it is easily seen that they may all be regarded as natural extensions of the relevant 
dichotomy of Hall (with the proviso that if there exist varieties which do not satisfy ( $i$ ) of Proposition 1 , the extension will not be quite so natural in this case). Now Hall [7; p. 602] commented that his dichotomy distinguished the properties 'nilpotent', 'locally polycyclic' and 'locally finitely related'. We note that the dichotomies described in Proposition 1 and in (ii) of Theorem A distinguish, for a soluble variety $\underline{V}$, the properties 'all torsion-free groups of $\underline{\underline{V}}$ are locally polycyclic (locally finitely related)' and 'all groups of $\underline{\underline{V}}$ are locally polycyclic (locally finitely related)', respectively. Also, the dichotomies described in (i) of Theorem $A$ and in Proposition 2 distinguish the properties 'all torsion-free groups of $\underline{\underline{V}}$ are nilpotent' and 'all groups of $\underline{\underline{V}}$ are nilpotent', respectively. Thus these results give a reasonably complete extension of Hall's dichotomy.

In order to state a generalization of Theorem $A$ we shall require a definition. We shall say that a variety is an sc-variety if it is a subvariety of a product of varieties each of which is either soluble or Cross. Thus the class of SC-varieties is precisely the smallest class of varieties which contains all soluble varieties and all Cross varieties and which is closed under the product operation and the operation of taking subvarieties.

As a step towards extending Theorem A (and as an interesting parallel to (ii) of Theorem A), we shall prove

THEOREM B. Let $\mathrm{V}$ be an SC-variety. If ( $\operatorname{var} G) \underline{\underline{\underline{A}}} \neq \underline{\underline{\mathrm{V}}}$ for all nonabelian finite simple groups $G$, then there exist natural numbers $I$ and $n$ such that $\underline{\underline{\mathrm{V}}} \leq \underline{\underline{\mathrm{A}}}^{{ }^{2}} \underline{\mathrm{B}}_{n}$.

By a straightforward induction on the minimal number of varieties required when we express $\underline{\underline{V}}$ as a subvariety of a product of soluble and Cross varieties, it is easily seen that it will be sufficient to prove

LEMMA B. Let $\underline{\underline{\mathrm{S}}}$ be a Cross variety and suppose that $\underline{\underline{\mathrm{V}}} \leq \underline{\underline{\mathrm{SA}}}^{2}$ for some natural number 2 . If $(\operatorname{var} G) \underline{\underline{A}} \$ \underline{\underline{V}}$ for all nonabelian finite simple groups $G$, then there exist natural numbers $m$ and $n$ such that $\underline{\underline{\mathrm{V}}} \leq \underline{\underline{\mathrm{A}}} \stackrel{\mathrm{m}}{\mathrm{B}}_{n}$. 
Finally, we come to our generalization of Theorem A.

THEOREM C. Let $\underline{\underline{\mathrm{V}}}$ be an SC-variety.

(i) If $\underline{\underline{\mathrm{AA}}} \not \underline{\underline{\mathrm{V}}}$ for all primes $p$, then there exist natural numbers $n$ and $c$ such that $\underline{\underline{V}} \leq \underset{n}{n} \frac{N}{N}$.

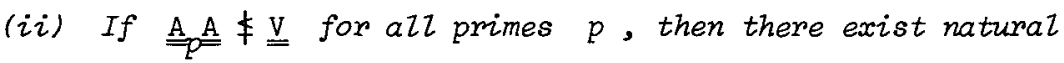
numbers $n$ and $c$ such that $\underline{\underline{\mathrm{V}}} \leq \underline{\underline{\mathrm{N}}} \mathrm{e}_{\mathrm{B}} \mathrm{B}_{n}$.

(iii) If $\underline{\underline{\mathrm{AA}}} p \neq \underline{\underline{\mathrm{V}}}$ and $\underline{\underline{\mathrm{A}}} \underline{\underline{\mathrm{A}}} \neq \underline{\underline{\mathrm{V}}}$ for all primes $p$, then there exist a nilpotent variety $\underline{\underline{\mathrm{N}}}$ and a variety $\underline{\underline{\mathrm{P}}}$ of finite exponent such that $\underline{\underline{V}}=\underline{\underline{N}} \vee \underline{\underline{P}}$.

We note, firstly, that ( $i i i$ ) is an immediate consequence of (i) and (ii) and Lemma A. Also, (ii) follows immediately from Theorem B and (ii) of Theorem A. Hence it only remains to prove $(i)$, and by an induction similar to that in the reduction of Theorem B to Lema B, it will suffice to prove

LEMMA C. Let $\underline{\underline{\mathrm{V}}}$ be an SC-variety contained in $\underline{\underline{\mathrm{AB}}}$ for some natural number $m$. If $\underset{\mathrm{AA}}{\mathrm{Q}} \neq \underline{\mathrm{V}}$ for all primes $p$, then there exist natural numbers $n$ and $c$ such that $\underline{\underline{\mathrm{V}}} \leq \underline{\mathrm{B}}_{n} \underline{\underline{N}}_{C}$.

\section{Preliminary results}

In many ways, our results were motivated by the classification of metabelian varieties of exponent zero, due to Kovács and Newman and given as 6.1.1 and 6.1.2 of Bryce [2]. We quote these here for convenience. (We call a variety torsion-free if its free groups are torsion-free.)

PROPOSITION 3. Let $\underline{\mathrm{V}}$ be a proper subvariety of $\underline{\underline{\mathrm{A}}}^{2}$ and suppose that $\underline{\underline{V}}$ is not of finite exponent. Then there exist a unique torsion-free variety $\underline{\underline{T}}$ and a unique natural number $n$, such that

$$
\underline{\underline{\mathrm{V}}}=\underline{\mathrm{T}} \vee \underline{\underline{A}} \underline{\underline{\underline{A}}} \vee \underline{\underline{P}}
$$

where $\underline{\underline{P}}$ has finite exponent.

PROPOSITION 4. The varieties $\stackrel{\mathrm{N}}{=} \underline{\underline{A}}_{S} \wedge \underline{\underline{A}}^{2}$ are torsion-free and 
join-irreducible. Every torsion-free proper subvariety of $\underline{\underline{A}}^{2}$ can be uniquely expressed as an irredundant join of some of these torsion-free join-irreducibles.

The following lemma, which is a consequence of Proposition 3, is proved in $[3 ; \mathrm{p} .97]$.

LEMMA 1. Let $n$ and $l$ be natural numbers and $\underline{\underline{V}}$ a variety such that $\stackrel{\mathrm{A} A}{\Rightarrow} \neq \mathrm{V}$ for every prime divisor $p$ of $n$. Then there exists a natural number $m$ (depending only on $n, l$ and $\underline{\underline{\mathrm{V}}}$ ) such that $\underline{\mathrm{B}}_{m}(G)$ centralises $H$ whenever $H \leq G$ and $H \in \underline{\underline{A}}^{2} \wedge \underline{B}_{n}$.

The first of the next two lemmas, which appears as Lerma 6 of Smel'kin [19] (and which is proved in [4]), may be regarded as the 'torsion-free counterpart' of a theorem of Hall [6] (as sharpened by Stewart [20]). The second is a varietal corollary which follows from the first by an easy induction on the solubility length of the variety, using the information obtainable from Proposition 4 as a starting point. (If $N$ is a torsion-free nilpotent group and $L \leq N$, we denote the isolator of $L$ in $N$ by $\left.I_{N}(L).\right)$

LEMMA 2. Suppose that $N$ is a torsion-free nilpotent group of class $c$, that $N$ is a normal subgroup of a group $G$ and that $G / I_{N}\left(N^{\prime}\right)$ is nilpotent of class $d$. Then $G$ is nilpotent of class at most $c d+(c-1)(d-1)$.

LEMMA 3. Let $\underline{\underline{\mathrm{V}}}$ be a variety such that $\underline{\underline{\mathrm{A}}}^{2} \neq \underline{\underline{\mathrm{V}}}$, and let $l$ be a natural nomber. Then there is a bound on the nilpotency class of the torsion-free nilpotent groups of $\underline{\underline{A}}^{2} \wedge \underline{\underline{V}}$.

We also recall the concept of the verbal Fitting subgroup, introduced in [3], which is defined as the product of the nilpotent verbal subgroups of a group. Since, as we noted in [3], in a relatively free group of infinite rank the centraliser of a verbal subgroup is verbal, the verbal Fitting subgroup contains its centraliser in this case.

It is well known that a finitely generated nilpotent group has a torsion-free subgroup of finite index. We shall next prove a 
generalization of this result which applies to arbitrary nilpotent groups. If. $G$ is an arbitrary group, we denote the $k$-th term of the upper central series of $G$ by $Z_{k}(G)$ (so that $Z_{1}(G)$ is the centre of $G$ ).

LEMMA 4. Let $N$ be a nilpotent group of class $c$ whose torsion subgroup $T$ is of finite exponent, $m$ say. Then, for some natural number $k, \underline{B}_{k}(N)$ is torsion-free.

Proof. Step 1. Suppose firstly that $c \leq 2$ and $N^{\prime} \leq T$. Let $a, b \in N$. Then, since $N$ has class at most 2 ,

$$
(a b)^{n}=a^{n} b^{n}[b, a]^{n(n-1) / 2}
$$

for any natural number $n$. Hence, since $[b, a] \in N^{\prime}$, which has exponent dividing $m$, the map of $N$ defined by $\tau: a \mapsto a^{2 m}$ will be an endomorphism of $N$ with kernel $T$ and image $\underline{B}_{2 m}(N)$. Hence B $_{2 m}(N) \cong N / T$ which is torsion-free and so the lemma is proved in this case.

Step 2. Suppose now that $N^{\prime} \leq T$ and $N$ has class $c$. Step 1 enables us to commence an inductive proof and so we may suppose that $\underline{\mathrm{B}}_{2}\left(N / \underline{\underline{N}}_{C-1}(N)\right)$, that is $\underline{\underline{\mathrm{B}}}_{2}(N) \cdot \underline{\underline{\mathrm{N}}}_{C-1}(N) / \underline{\underline{N}}_{C-1}(N)$, is torsion-free for some natural number 2 . Thus

$$
\begin{aligned}
&\left(\underline{\mathrm{B}}_{Z}(N)\right)^{\prime} \leq \underline{\underline{B}}_{Z}(N) \cap N^{\prime} \leq \underline{\underline{B}}_{Z}(N) \cap T \leq \underline{\underline{B}}_{2}(N) \cap \underline{\underline{N}}_{C-1}(N) \\
&\left.\leq \underline{\underline{B}}_{Z}(N) \cap Z Z_{1}(N) \leq Z_{1} \underline{\underline{B}}_{Z}(N)\right) .
\end{aligned}
$$

Hence $\underline{\underline{B}}_{Z}(N)$ has class at most 2 and $\left(\underline{\underline{B}}_{Z}(N)\right)^{\prime} \leq \underline{\underline{B}}_{Z}(N) \cap T$, the torsion subgroup of $\underline{\underline{B}}_{\ell}(N)$. We may now apply Step 1 to show that, for some $k \in N$ (the set of natural numbers), $\underline{B}_{k}\left(\underline{B}_{l}(N)\right)$, and so $\underline{B}_{k l}(N)$, is torsion-free, completing the proof of the lemma in this case.

Step 3. We claim that, if $N$ satisfies the conditions of the lemma, then the torsion subgroup of $N / Z_{C-1}(N)$ has finite exponent dividing $m$. For, let $a \in N, n \in \mathbb{N}$ and suppose that $a^{n} \in Z_{c-1}(N)$. Then, for all sequences $b_{1}, \ldots, b_{c-1}$ of elements of $N,\left[a^{n}, b_{1}, \ldots, b_{c-1}\right]=e$. 
Thus $\left[a, b_{1}, \ldots, b_{c-1}\right]^{n}=e$ and so $\left[a, b_{1}, \ldots, b_{c-1}\right] \in T$. Hence

$$
\left[a, b_{1}, \ldots, b_{c-1}\right]^{m}=\left[a^{m}, b_{1}, \ldots, b_{c-1}\right]=e
$$

and so $a^{m} \in Z_{c-1}(N)$, proving our claim.

Step 4. We are now ready to complete the proof of the lemma by induction on $c$. The case $c=1$ was included in Step 1 . As the torsion subgroup $T \cap Z_{C-1}(N)$ of $Z_{C-1}(N)$ is certainly of finite exponent, we may apply the induction hypothesis to show that there is a natural number $j$ such that $\underline{\underline{B}}_{j}\left(Z_{c-1}(N)\right)$ is torsion-free. Now $\underline{\underline{B}}_{j}\left(Z_{c-1}(N)\right) \leq N$ and, since by step 3 the torsion subgroup of $N / Z_{C-1}(N)$ has finite exponent, so also does that of $N / \underline{\underline{B}}_{j}\left(Z_{c-1}(N)\right)$. Since $N^{\prime} \leq Z_{c-1}(N),\left(N / \underline{\underline{B}}_{j}\left(Z_{c-1}(N)\right)\right)^{\prime}$ has finite exponent. Thus we may apply step 2 to show that, for some natural number $i, \underline{B}_{i}\left(N / \underline{\underline{B}}_{j}\left(z_{c-1}(N)\right)\right)$, that is

$$
\underline{\mathrm{B}}_{i}(N) \cdot \underline{\mathrm{B}}_{j}\left(Z_{c-1}(N)\right) / \mathrm{B}_{j}\left(Z_{c-1}(N)\right),
$$

is torsion-free. Since $\stackrel{B}{B}_{j}\left(Z_{c-1}(N)\right)$ is torsion-free, this implies that so is $\underline{B}_{i}(N) \cdot \underline{\underline{B}}_{j}\left(Z_{e-1}(N)\right)$, and therefore also $\underline{B}_{i}(N)$, completing the proof of the lemma.

The following lemma was inspired by Lemma 2.2 of Neumann [15] and may be regarded as an 'upside-down' version of that result.

LEMMA 5. Let $G$ be a group which generates the variety $\underline{V}$ and let $\Delta$ be an infinite set of natural numbers. Let $\left\{H_{n} \mid n \in \Delta\right\}$ be a set of groups with the following property:

there is a normal subgroup $L_{n}$ of $H_{n}$ and an epimorphism $\lambda_{n}: L_{n} \rightarrow G$ and an element $h_{n}$ of $H_{n}$, such that, if $n_{n}$ denotes the restriction to $L_{n}$ of the inner automorphism induced by $h_{n}$, then the epimorphisms $\eta_{n}^{i} \lambda_{n}: L_{n} \rightarrow G$ $(0 \leq i \leq n)$ are independent in the sense that, whenever 
$g_{0}, \ldots, g_{n}$ is a sequence of elements of $G$, there exists an

element $l$ of $L_{n}$ such that

$$
2 n_{n}^{i} \lambda_{n}=g_{i} \quad(0 \leq i \leq n) .
$$

Then $\underline{\underline{\mathrm{VA}}} \leq \operatorname{var}\left\{H_{n} \mid n \in \Delta\right\}$.

(Note: The purpose of this rather unwieldy looking lemma is to deal with the case where $L_{n}$ has a normal subgroup $K_{n}$ - the kernel of $\lambda_{n}$ such that $L_{n} \int_{i=0}^{n} K_{n}^{h_{n}^{i}}$ is isomorphic to a direct power of $G$. The epimorphisms $\eta_{n}^{i} \lambda_{n}$ give the canonical epimorphisms onto the direct factors and their independence expresses the fact that the above quotient is indeed a direct power.)

Proof. Let $w=w\left(x_{1}, \ldots, x_{p}\right)$ be a word which is not a law of $\underline{\underline{V A}}$. It will evidently suffice to show, for some $n \in \Delta$, that $w$ is not a law of $H_{n}$. Now the independence of the epimorphisms $\eta_{n}^{i} \lambda_{n}$ implies that $h_{n}$ has order at least $n+1$. Since $\Delta$ is an infinite set, $\underline{\underline{\mathrm{A}}} \leq \operatorname{var}\left\{H_{n} \mid n \in \Delta\right\}$ and we may suppose that $w \in X^{\prime}$.

Let $C=g P(c)$ be an infinite cyclic group. Then, by 22.44 of [14], $G$ wr $C$ generates VA. Hence $w$ is not a law of $G$ wr $C$ and therefore there is a homomorphism $\xi: X \rightarrow G$ wr $C$ such that $w \xi \neq e$. Suppose that $\bar{\xi}: x_{i} \mapsto c^{s(i)} \phi_{i}$ where $s(i)$ is an integer and $\phi_{i}$ is an element of the base group of $G$ wr $C$. Define $\delta: X \rightarrow X$ by

$$
\delta: x_{i} \mapsto x_{1}^{s(i)} x_{i+1}
$$

and $\xi^{\prime}: X \rightarrow G$ wr $C$ by

$$
\xi^{\prime}: x_{1} \mapsto c, x_{i} \mapsto \phi_{i-1}(i \neq 1) .
$$

Then $\delta \xi^{\prime}=\xi$ and so $w \delta \xi^{\prime} \neq e$. Now, by 22.34 of [14], 


$$
\begin{aligned}
w \delta=w\left(x_{1}, \ldots, x_{r}\right) \delta=w\left(x_{1}^{s(1)} x_{2}, \ldots, x_{1}^{s(r)} x_{r+1}\right) & \\
& =w\left(x_{1}^{s(1)}, \ldots, x_{1}^{s(r)}\right) \prod_{j=1}^{t} x_{i(j)+1}^{\varepsilon(j) x_{1}^{k(j)}}
\end{aligned}
$$

where $\varepsilon(j)= \pm 1,1 \leq i(j) \leq r, k(j)$ is an integer. But, since $w \in X^{\prime}$,

$$
w\left(x_{1}^{s(1)}, \ldots, x_{1}^{s(r)}\right)=e
$$

Hence

$$
w \delta \xi^{\prime}=\prod_{j=1}^{t} \phi_{i(j)}^{\varepsilon(j) e^{k(j)}} \neq e
$$

and so, for some $e^{m} \in C$,

$$
\prod_{j=1}^{t} \phi_{i(j)}^{\varepsilon(j) c^{k(j)}}\left(c^{m}\right)=\prod_{j=1}^{t} \phi_{i(j)}^{\varepsilon(j)}\left(c^{m-k(j)}\right) \neq e .
$$

Denote $-\min _{j}(\{k(j)\})$ by $a$ and $\max _{j}(\{k(j)\})$ by $b$ and let $n$ be an element of $\Delta$ such that $n \geq a+b$. We shall show that $w$ is not a law of $H_{n}$; to ease notation we shall drop the subscript $n$. For each ordered pair of integers $u, v$ satisfying $1 \leq u \leq r, 0 \leq v \leq n$ define $g_{u, v}=\Phi_{u}\left(c^{m+a-v}\right)$. Then, using the independence condition on the $n^{v} \lambda$, there exist, for each $u$, elements $\tau_{u}$ in $L$ such that

$$
\tau_{u} \eta^{v} \lambda=g_{u, v}=\phi_{u}\left(c^{m+a-v}\right)
$$

Define $\tau: X \rightarrow H$ by

$$
\begin{array}{rll}
\tau & : x_{1} \mapsto h & \\
& : x_{i} \mapsto z_{i-1} & (2 \leq i \leq x+1) \\
& : x_{i} \mapsto e & \text { otherwise. }
\end{array}
$$

Then 


$$
\omega \delta \tau=\left(\prod_{j=1}^{t} \begin{array}{c}
\varepsilon(j) x_{1}^{k(j)} \\
x_{i(j)+1}
\end{array}\right) \tau=\prod_{j=1}^{t} i_{i(j)}^{\varepsilon(j) h^{k(j)}}
$$

Hence

$$
\begin{aligned}
& w \delta \operatorname{\tau n}^{a} \lambda=\left(\prod_{j=1}^{t} z_{i(j)}^{\varepsilon(j) h^{k(j)}}\right) n^{a_{\lambda}} \\
& =\prod_{j=1}^{t}\left(z_{i(j)^{n^{a+k(j)}} \lambda}\right)^{\varepsilon(j)} \\
& =\prod_{j=1}^{t} \phi_{i(j)}^{\varepsilon(j)}\left(c^{m+a-a-k(j)}\right) \\
& =\omega \delta \xi^{\prime}\left(c^{m}\right) \neq e \text {. }
\end{aligned}
$$

Thus $w \delta \tau \neq e$ and so $w$ is not a law of $H$, completing the proof.

For the proof of Lemma $B$, we shall also need the following lemma - a varietal analogue to Theorem 5.3 of Neumann [13].

LEMHA 6. If $\underline{\underline{P}}$ is a locally finite variety, then [ $\underline{\underline{E}}$, $\underline{\underline{P}}] \leq \underline{\underline{B}} \underline{\underline{A}}$ for some natural number $n$.

Proof. Let the exponent of $\underline{P}$ be $m$. By a theorem of Bryant [1], $\stackrel{B}{=} \vee \underline{\underline{A}}$ is finitely based; suppose that $w=w\left(x_{1}, \ldots, x_{k}\right)$ is a basis for its laws. Denote $F_{k}([\underline{E}, \underline{\underline{P}}])$ by $F$ and let $y_{1}, \ldots, y_{k}$ be a free generating set of $F$. Then $F / \underline{\underline{P}}(F)$ is a finitely generated group of $\underline{\underline{\mathrm{P}}}$ and so is finite. Hence $\underline{\underline{P}}(F)$ is a central subgroup of finite index in $F$ and so, by Theorem 5.3 of [13], $F^{\prime}$ is finite. In particular, $\stackrel{B}{m}_{m}(E) \cap F^{\prime}$ is of finite exponent, $Z$ say. Thus, since $w\left(y_{1}, \ldots, y_{k}\right) \in \underline{\mathrm{B}}(F) \cap F^{\prime}$, $w\left(y_{1}, \ldots, y_{k}\right)^{2}=e$.

Denote $F_{\infty}([\underline{\underline{E}}, \underline{\underline{P}}])$ by $H$ and let $y_{1}, \ldots, y_{k}, \ldots$ be a free generating set of $H$. Then the relation $w\left(y_{1}, \ldots, y_{k}\right)^{2}=e$ holds in $H$ also. But the word $w$ is a basis for the laws of the variety $\underline{\underline{A}} \vee \underline{B}$ and so $(\underline{\underline{A}} \vee \stackrel{\mathrm{B}}{\Rightarrow})(H) \leq H^{\prime} \cap \stackrel{\mathrm{B}}{\mathrm{B}_{m}}(H)$ is generated by all endomorphic images of $w\left(y_{1}, \ldots, y_{k}\right)$. Evidently each of these images is of order dividing $l$. 
Since $H^{\prime} \cap \underline{\underline{B}}_{m}(H)$ is abelian, even central, (A $\left.\vee \underline{\mathrm{B}}_{m}\right)(H)$ is thus of finite exponent dividing 2 . Therefore $H \in \underline{\underline{B}}_{2}\left(\underline{A} \vee \underline{\underline{B}}=\leq \underline{\underline{B}}_{2} \underline{B}_{m} \stackrel{A}{=} \leq \underline{\underline{B}}_{2 m} \stackrel{A}{m}\right.$ and so $[\underline{\underline{E}}, \underline{\underline{P}}] \leq \underline{\underline{B}} \underline{\underline{A}}$ where $n=2 m$.

The proof of the following lemma is not difficult and we shall omit it.

LEMMA 7. Let $G$ be a group with torsion-free derived group. Then $\operatorname{var} G$ is either abelian or torsion-free.

Finally, we formalize a technique introduced in [3; p. 99] for finding minimal counterexamples in results of the type we consider.

LEMI'A 8. Suppose that $\Lambda$ is a set of finitely based varieties and that $\Omega=\{\underline{\underline{U}} \mid \underline{\underline{U}} \neq \underline{\underline{V}}$ for all $\underline{\underline{V}} \in \Lambda\}$. Then each element of $\Omega$ contains a minimal element of $\Omega$.

\section{Proof of Theorem $A$}

3.1 Proof of $(i)$. For a proof by contradiction, we suppose that $\underline{V}$ is a counterexample to $(i)$ of Theorem $A$. Since $\underline{\underline{V}}$ is soluble, the condition that $\underline{\mathrm{V}} \leq \underline{\mathrm{B}} \underset{n}{\mathrm{~N}} \mathrm{C}$ for some natural numbers $n$ and $c$ is equivalent to the condition that $\underline{\underline{V}} \leq \underline{A}_{k}^{2} \underline{\underline{N}}_{C}$ for some natural numbers $k, Z$ and $c$. However, by 34.14 of [14] and repeated application of Theorem 3.1 of Higman [9], every variety in the set $\left\{A_{k}^{2} \stackrel{N}{c}_{c} \mid k, z, c \in N\right\}$ is finitely based. Thus an application of Lemma 8 shows that $V$ contains a subvariety which is a minimal counterexample. We may suppose without loss of generality that $\underline{\underline{V}}$ is this minimal counterexample.

Denote $F_{\infty}(\underline{\mathrm{V}})$ by $F$ and let $T$ be a verbal torsion subgroup of $F$. Since $T$ is soluble, if it were non-trivial, there would be a non-trivial abelian subgroup $A$ of $T$, verbal in $T$ and so verbal in $F$. Also $A$ would have a fully invariant subgroup, $B$, of finite exponent and so $B$ would be verbal in $F$ (for example, since $A$ must have a non-trivial $p$-element for some prime $P$, we could take $B$ as the subgroup of all elements of order dividing $p$ ). Then $F / B$ would generate a proper subvariety of $\underline{\underline{V}}$ and it is immediate that $\underline{\underline{V}}$ could not be a minimal 
counterexample in this case. Thus $T$ is trivial. In particular, every nilpotent verbal subgroup of $F$ is torsion-free. Since the conditions of the theorem imply that $\underline{\underline{A}}^{2} \neq \underline{\underline{V}}$, Lemma 3 shows that $\underline{\underline{V}}$ has a bound on the class of its torsion-free nilpotent groups. Thus the verbal Fitting subgroup of $F, N$ say, is nilpotent and torsion-free.

Suppose that $F / N$ is non-trivial. Then there is a verbal subgroup, $A$ say, of $F$ such that $A / N$ is abelian and non-trivial. Since $A^{\prime} \leq N$ : $A / I_{N}\left(N^{\prime}\right)$ is a metabelian group with torsion-free derived group. By Lemma 7, $\operatorname{var}\left(A / I_{N}\left(N^{\prime}\right)\right)$ is either abelian or torsion-free. The conditions of the theorem, together with Proposition 4, however, show that the torsion-free metabelian subvarieties of $\underline{\underline{V}}$ are nilpotent. Hence, in either case, $A / I_{N}\left(N^{\prime}\right)$ is nilpotent. Thus, by Lemma $2, A$ is nilpotent. Then, since $A$ is verbal in $F, A \leq N$, contradicting the assumption that $A / N$ is non-trivial. So $F / N$ is trivial and $\underline{V}$ is nilpotent and so certainly not a counterexample as we supposed. The proof of ( $i$ ) is complete.

3.2 Proof of ( $i i)$. The proof is again by contradiction. Since, by Theorem 3.1 of Higman [9] all varieties of the set $\left\{\underline{N}_{c} B \mid c, n \in N\right\}$ are finitely based, we may, as in 3.1 suppose that $\underline{V}$ is a minimal counterexample to $(i i)$ of Theorem A. Denote $F_{\infty}(\underline{V})$ by $F$. Then the proof splits into two cases:

a) $E$ has a non-trivial, verbal, torsion subgroup.

In this case, as in 3.1, $F$ has a non-trivial, elementary abelian, verbal subgroup, $A$ say. Then $F / A$ generates a proper subvariety of $\underline{V}$ and so $F / A \in \underline{\underline{N}} \underset{c}{B} n$ for some natural numbers $c, n$. Since $\stackrel{A}{\Rightarrow} \equiv \underline{\underline{V}}$ for all primes $p$, Lemma $I$ shows that $\underline{B}_{m}(F)$ centralises $A$ for some natural number $m$. Now

$$
\left(\underline{\mathrm{B}}_{m}(F) \cdot A \cap \underline{\underline{\mathrm{B}}_{n}}(F) \cdot A\right) / A \leq \underline{\mathrm{B}_{n}}(F) \cdot A / A=\underline{\underline{\mathrm{B}}}_{n}(F / A),
$$

which is nilpotent. Thus, since $A$ is central in $\mathrm{B}_{m}(F) \cdot A$, and so in $\left.\underline{B}_{m}(F) \cdot A \cap \underline{B}_{n}(F) \cdot A\right)$, the latter subgroup is also nilpotent. Since 
$E /\left(\underline{B}_{m}(F) \cdot A \cap \underline{B}_{n}(F) \cdot A\right)$ is of finite exponent, $\underline{\mathrm{V}}$ cannot be a counterexample as we supposed, which completes the proof in this case.

b) $F$ has no non-trivial, verbal, torsion subgroup.

In this case, as in 3.1, the verbal Fitting subgroup, $N$ say, of $F$ is nilpotent and torsion-free. Since $F$ is soluble $N$ is non-trivial and so $F / N$ generates a proper subvariety of $\underline{\underline{\mathrm{V}}}$; suppose that $F / N \in \underline{\underline{\mathrm{N}}} \underline{\underline{B}}$ $(c, n \in N)$. Let $A$ be the subgroup of $F$ defined by $A / N=Z_{1}\left(\underline{B}_{n}(F / N)\right)$. Then $A$ is verbal in $F$ and since $\stackrel{B}{n}_{n}(F / N)$ is nilpotent, $A / N$ is non-trivial. Let $I$ denote $I_{N}\left(N^{\prime}\right)$. Then $A / I$ is metabelian and the derived group of $A / I$ is torsion-free. Hence, by Lemma 7 and Proposition 4, $A / I \in \underset{N}{\mathbb{N}} d_{m}$ for some natural numbers $d, m$. Put $\underset{m}{\mathrm{~B}}(A / I)=B / I$. Then $B$ is verbal in $F$, and $B / I$, and so $B N / I$, is nilpotent. Hence, by Lemma 2, $B N$ is nilpotent. Thus $B \leq N$ and so $A / N \in \stackrel{B}{=m}$. But $A / N$ was defined as $Z_{1}\left(\underline{B}_{n}(F / N)\right)$, so by Corollary 1.62 of $[18], \underline{B}_{n}(F / N)$ has finite exponent. Thus $F / N$ has finite exponent, $\underline{\underline{V}}$ cannot be a counterexample in this case either, and the proof is complete.

3.3 Proof of Lemma A. Denote $F_{\infty}(\underline{\underline{V}})$ by $F, \underline{B}_{n}(F)$ by $B, \underline{\underline{N}}_{c}(F)$ by $N$, and the torsion subgroup of the nilpotent group $B$ by $T$. Since $B \cap N \in \underline{\mathrm{B}}_{n}, T \geq B \cap N$. Thus $T / B \cap N=T / T \cap N \cong T N / N$. But $F / N$, as a nilpotent free group, has maximum condition on its fully invariant subgroups (this follows from 34.24 of [14]); in particular, the torsion subgroup of $E / N$ has finite exponent. Thus $T N / N \cong T / B \cap N$ has finite exponent and so $T$ has finite exponent. Hence, by Lemma $4, B_{m}(B) \cap T=E$ for some natural number $m$. Therefore,

$$
\underline{\mathrm{B}_{m}}(B) \cap N=\underline{\mathrm{B}_{m}}(B) \cap(B \cap N) \leq \underline{\mathrm{B}}_{m}(B) \cap T=E .
$$

Since $\stackrel{\mathrm{B}}{\Rightarrow m}(B) \geq \stackrel{\mathrm{B}}{m n}_{m}(F)$ and $N=\underline{\underline{N}}_{c}(F), \underline{\underline{\mathrm{V}}}=\left(\underline{\mathrm{B}}_{m n} \wedge \underline{\underline{\mathrm{V}}}\right) \vee\left(\underline{\mathrm{N}}_{c} \wedge \underline{\underline{\mathrm{V}}}\right)$ and the proof is complete.

\section{Proof of Lemma B}

The proof is by contradiction; we suppose that $\underline{\underline{V}}$ is a 
counterexample to the lemma. By repeated application of Theorem 3.1 of Higman [9], every variety $\stackrel{m_{\mathrm{B}}}{=}$ is finitely based. Thus an application of Lemma 8 shows that $\underline{V}$ contains a subvariety which is a minimal counterexample and evidently we may suppose that $\underline{V}$ is this minimal counterexample. Denote $F_{\infty}(\underline{\underline{V}})$ by $F$ and let $y$ be a free generator of $F$.

Since $F \in \underline{\underline{S A}} Z$ and the Cross variety $\underline{\underline{S}}$ has only finitely many subvarieties, $\mathrm{A}^{l}(F)$ has a minimal verbal subgroup, $W$ say; evidently, $W$ is verbally simple and is verbal in $F$. Since $W \in \underline{\underline{S}}$ and subvarieties of Cross varieties are Cross, var $W$ can be generated by a finite group. Let $G$ be a finite group of least order generating $\operatorname{var} W$.

We claim that $G$ is simple. For, let $N \unlhd G$. Then $W \in \operatorname{var} G \leq(\operatorname{var} N)(\operatorname{var}(G / N))$. But since $W$ is verbally simple, the verbal subgroup of $W$ corresponding to $\operatorname{var}(G / N)$ is either trivial or $W$ itself. In the first case, $W \in \operatorname{var}(G / N)$ and so $N=E$, by the minimality of $G$; in the second case, $W \in \operatorname{var} N$ and so $N=G$, again by the minimality of $G$. Thus $G$ is simple. If $G$ is abelian, then $\mathrm{V} \leq \mathrm{A} \operatorname{var}(F / W) \leq \mathrm{AN} d \mathrm{~B}$ for some natural numbers $d$ and $m$, as $F / W$ generates a proper subvariety of $\underline{V}$, and by Theorem $A$ this contradicts the choice of $\underline{V}$ as a minimal counterexample. Hence $G$ is a non-abelian finite simple group. Denote the subvariety of $\operatorname{var} G$ generated by the proper sections of $G$ by $\underline{R}$.

Let $\Gamma$ be the set $\{N \mid N \unlhd W$ and $W / N \cong G\}$. We claim that ก $N=E$. Evidently, it will suffice to show that, if $e \neq w \in W$, then $N \in \Gamma$

there is a normal subgroup $N$ of $W$ such that $w \notin N$ and $W / N \cong G$. With this in mind, let $N$ be a normal subgroup of $W$ maximal with respect to avoiding $w$ and let $M$ denote the normal subgroup of $W$ generated by $w$ and $N$; the existence of $N$ is guaranteed by Zorn's lemma. Then $M / N$ is a chief factor of $W$ and $W / N$ is monolithic. Also $M / N$ is finite, since $W$ lies in the cross variety $S$ (cf. 52.21 of [14]). In order to show that $W / N \cong G$, we must firstly show that $M / N$ is non-abelian.

Since $\operatorname{var} W=\operatorname{var} G$ and $W$ is verbally simple, $\underline{\underline{R}}(W)=W$. Now 
$\underline{\mathrm{R}}$, being a Cross variety, is finitely based (see, for example, 52.12 of [14]); let the word $r\left(x_{1}, \ldots, x_{k}\right)$ be a basis for the laws of $\underline{\underline{R}}$. Since $w \in W=\underline{R}(W)$, there are elements $a_{i j}(1 \leq i \leq k, 1 \leq j \leq 2)$ of $W$ such that

$$
w=\prod_{1 \leq j \leq l} r\left(a_{1 j}, \ldots, a_{k j}\right)
$$

Let $Y=\operatorname{gp}\left(\left\{a_{i j} \mid 1 \leq i \leq k, 1 \leq j \leq \ell\right\}, M\right)$. Then $Y / M$ is a finitely generated group of the Cross variety $\underline{S}$ and therefore it is finite. Since $M / N$ is also finite, $\mathrm{Y} / N$ is finite. Now $w N \in \underline{\mathrm{R}}(Y / N) N M / N$ and $w N$ is non-trivial. Thus $\underline{\underline{R}}(Y / N) M / N$ is non-trivial. As Peter $M$. Neumann noted ([16], p. 77), the proof of Lemma 3.2 of Oates [17] shows that the R-subgroups of the finite groups in var $G$ are direct powers of $G$ (the additional hypotheses of Oates being unnecessary). Hence $\underline{\underline{R}}(Y / N) N M / N$, being a normal subgroup of $\underline{\underline{R}}(Y / N)$, is also such a direct power. In particular, $M / N$ is non-abelian. By Theorem 4 of Kovács and Newman [10], $W / N$ is isomorphic to a section of $G$. Since $\underline{\underline{R}}(W / N)=\underline{R}(W) N / N=W / N$ is non-trivial, this section cannot be a group of $\underline{\underline{\mathrm{R}}}$. Hence $W / N \cong G$, as required.

The proof now splits into two cases. Suppose, firstly, that for some $n \in N, N^{y^{n}}=N$ for all $N \in \Gamma$. Then $y^{n}$ normalises $N$ and, if $m$ is the exponent of Aut $G, y^{n m}$ centralises $W / N$, for all $N \in \Gamma$. Hence $\left[W, y^{n m}\right] \leq \bigcap_{N \in \Gamma} N=E$ and so $y^{n m} \in C_{F}(W)$, the centralizer of $W$ in $F$. But $\mathcal{C}_{F}(W)$ is verbal in $F$ and so, since $y$ is a free generator of $F$,

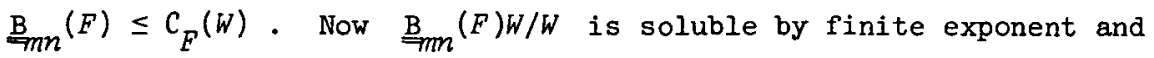
therefore so also is $\stackrel{\mathrm{B}}{m m}_{m}(F) /\left(\mathrm{B}_{m m}(F) \cap W\right)$. But we have shown that $\mathrm{B}_{m n}(F) \cap W$ is central in $\stackrel{\mathrm{B}}{7 m}^{(F)}$. Thus $\mathrm{B}_{m n}(F)$, and so $F$ itself, is soluble by finite exponent, which contradicts the choice of $\underline{V}$ as a minimal counterexample.

Thus we may reject the hypothesis in the first case and suppose that, for all $n \in \mathbb{N}$, there is an $N_{n} \in \Gamma$ such that $N_{n}^{y^{n !}} \neq N_{n}$. In preparation for an application of Lemma 5, we will show that 
$W \prod_{0 \leq i \leq n} M_{n}^{x^{i}} \cong G^{n+1} \quad$ (that is, the direct product of $n+1$ copies of $G$ ). Since $N_{n}^{y^{n !}} \neq N_{n}, \quad N_{n}^{y^{i}} \neq N_{n}^{y^{j}}$ whenever $0 \leq i<j \leq n$. Now, as

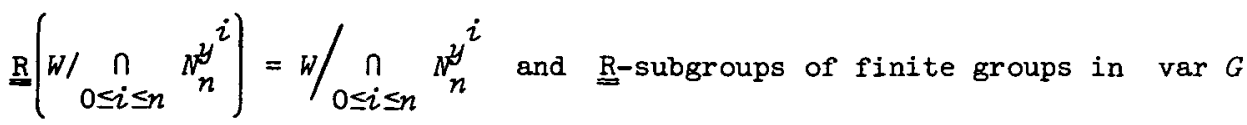
are direct powers of $G, W / \prod_{0 \leq i \leq n} N_{n}^{y^{i}}$ is such a direct power. Also, $w / \prod_{0 \leq i \leq n} N_{n}^{y^{i}}$ evidently has order at most $|G|^{n+1}$ and has at least $n+1$ distinct maximal normal subgroups. Thus $W / \bigcap_{0 \leq i \leq n} w_{n}^{y^{i}} \cong G^{n+1}$, as required. Transferring from the notation of Lemma 5, we let $\Delta=\mathrm{N}, H_{n}=F$, $h_{n}=y^{-1}, L_{n}=W$, and let $\delta_{n}$ be the homomorphism $\delta_{n}: W \rightarrow G$ with kernel $N_{n}$. Then $\eta_{n}$ is the restriction to $W$ of the inner automorphism of $F$ induced by $y^{-1}$ and the kernel of $n_{n}^{i_{\delta}}$ is $M_{n}^{y^{i}} \quad(0 \leq i \leq n)$. Let $\pi_{0}, \ldots, \pi_{i}, \ldots, \pi_{n}$ be the canonical projections $\pi_{i}: G^{n+1} \rightarrow G$ which define $G^{n+1}$. It is a defining property of (cartesian) products that, given the homomorphisms $\delta_{n}, n_{n} \delta_{n}, \ldots, n_{n}^{n} \delta_{n}: W \rightarrow G$, there is a unique homomorphism $\theta: W \rightarrow G^{n+1}$ such that $\theta \pi_{i}=\eta_{n}^{i} \delta_{n} \quad(0 \leq i \leq n)$ and $\operatorname{ker} \theta=\bigcap_{0 \leq i \leq n} \operatorname{ker} \eta_{n}^{i} \delta_{n}$. Then

$$
|w \theta|=\left|w / \prod_{0 \leq i \leq n} \operatorname{kern}_{n}^{i} \delta_{n}\right|=\left|w / \prod_{0 \leq i \leq n} N_{n}^{y^{i}}\right|=\left|G^{n+1}\right|
$$

and therefore, as $G^{n+1}$ is finite, $W \theta \cong G^{n+1}$. Thus, if $g_{0}, \ldots, g_{n} \in G$, we may take an element $h$ of $G^{n+1}$ such that

$$
h \pi_{i}=g_{i} \quad(0 \leq i \leq n)
$$

and then an element $v$ of $W$ such that $v \theta=h$, so that 


$$
v n_{n} \delta_{n}=v \theta \pi_{i}=g_{i} \quad(0 \leq i \leq n) .
$$

Hence the requirements of Lemma 5 are satisfied and so ( $\operatorname{var} G) \underline{A} \leq \underline{V}$, contradicting the hypothesis of the lemma and completing the proof.

\section{Proof of Lernma $C$}

The proof is by contradiction; we suppose that $\underline{V}$ is a counterexample to the lemma. By Lemma $A$, a subvariety of $\underline{\underline{V}}$ satisfies the conclusion of the lemma if (and only if) it is a subvariety of one of the varieties of the set $\left\{\mathbb{N}_{c} \vee \mathrm{B}_{n} \mid c, n \in \mathbb{N}\right\}$. Since, by a theorem of Bryant [1], every variety of this set is finitely based, we may apply Lemma 8 and suppose that $\underline{\underline{V}}$ is a minimal counterexample.

Let $F$ denote $F_{\infty}(\underline{\mathrm{V}})$ and $B$ denote $\underline{B}_{m}(F)$. Now the torsion subgroup of $B$ is fully invariant in $B$ and so verbal in $F$; if it were non-trivial, it would give rise to a non-trivial elementary abelian verbal subgroup of $F$. In this case, as in $3.1, \underline{\underline{V}}$ could not be a minimal counterexample. Hence $B$ is torsion-free. Let $x \in F$. Then, if $H=g p(B, x), H \in \underline{\underline{A A}}$ and $H^{\prime} \leq B$ is torsion-free. Thus by Lemma 7 , $\operatorname{var} H$ is a torsion-free subvariety of $\stackrel{\mathrm{AA}}{=}$; now the conditions of the lemma and Proposition 4 show that var $H=\underline{\underline{A}}$. This shows that every element of $F$ commutes with $B$; that is, $B \leq Z_{1}(F)$ and so $\underline{\underline{V}} \leq\left[\underline{\underline{E}}, \underline{\underline{V}} \wedge \stackrel{B}{g_{m}}\right]$. Since $\underline{\underline{\mathrm{V}}} \wedge \stackrel{\mathrm{B}}{\mathrm{B}_{m}}$ is an sC-variety of finite exponent, it is locally finite and so Lemma 6 shows that $\underline{\underline{V}} \leq \underline{B}$ A for some natural number $n$, contradicting the choice of $\underline{V}$ as a counterexample and completing the proof of the lemma.

\section{References}

[1] Roger M. Bryant, "On some varieties of groups", BuZZ. London Math. Soc. 1 (1969), 60-64.

[2] R.A. Bryce, "Metabelian groups and varieties", Phizos. Trans. Roy. Soc. London Ser. A 266 (1970), 281-355. 
[3] J.R.J. Groves, "On varieties of soluble groups", Bull. Austral. Math. Soc. 5 (1971), 95-109.

[4] J.R.J. Groves, "Varieties of soluble groups", (Ph.D. thesis, Australian National University, Canberra, 1971).

[5] P. Hall, "Finiteness conditions for soluble groups", Proc. London Math. Soc. (3) 4 (1954), 419-436.

[6] P. Hall, "Some sufficient conditions for a group to be nilpotent", IZZinois J. Math. 2 (1958), 787-801.

[7] P. Hall, "On the finiteness of certain soluble groups", Proc. London Math. Soc. (3) 9 (1959), 595-622.

[8] P. Hall, "The Frattini subgroups of finitely generated groups", Proc. London Math. Soc. (3) 11 (1961), 327-352.

[9] Graham Higman, "Some remarks on varieties of groups", Quart. J. Math. Oxford (2) 10 (1959), 165-178.

[10] L.G. Kovács and M.F. Newman, "Minimal verbal subgroups", Proc. Combridge Phizos. Soc. 62 (1966), 347-350.

[11] L.G. Kovács and M.F. Newman, "Just-non-Cross varieties", Proc. Internat. Conf. Theory of groups, Austral. Nat. Univ., Canberra, 1965, 221-223 (Gordon and Breach, New York, London, Paris, 1967).

[12] L.G. Kovács and M.F. Newman, "On non-Cross varieties of groups", $J$. Austral. Math. Soc. 12 (1971), 129-144.

[13] B.H. Neumann, "Groups with finite classes of conjugate elements", Proc. London Math. Soc. (3) 1 (1951), 178-187.

[14] Hanna Neumann, Varieties of groups (Ergebnisse der Mathematik und ihrer Grenzgebiete, Band 37. Springer-Verlag, Berlin, Heidelberg, New York, 1967).

[15] Peter M. Neumann, "On word subgroups of free groups", Arch. Math. 16 (1965), 6-21.

[16] Peter M. Neumann, "A note on the direct decomposability of relatively free groups", Quart. J. Math. Oxford (2) 19 (1968), 67-79. 
[17] Sheila Oates, "Identical relations in groups", J. London Math. Soc. $38(1963), 71-78$.

[18] Derek J.S. Robinson, Infinite soluble and nilpotent groups (Queen Mary College Mathematics Notes, Mathematics Department, Queen Mary College, London, N.D. [1968]).

[19] A.L. Smel'kin, "On soluble group varieties", Soviet Math. Dokz. 9 (1968), 100-103.

[20] A.G.R. Stewart, "On the class of certain nilpotent groups", Proc. Roy. Soc. London Ser. A 292 (1966), 374-379.

Department of Mathematics, Institute of Advanced Studies, Australian National University, Canberra, ACT.

Present address:

Department of Mathematics and Astronomy, University of Manitoba, Winnipeg,

Mani toba, Canada. 